\title{
Evaluation of vascular grafts based on polyvinyl alcohol cryogels
}

\author{
MARIA TERESA CONCONI ${ }^{1}$, LUCA BORGIO $^{1,2}$, ROSA DI LIDDO ${ }^{1}$, LEONARDO SARTORE $^{4}$, \\ DANIELE DALZOPPO ${ }^{1}$, PIETRO AMISTÀ ${ }^{3}$, SILVANO LORA ${ }^{2}$, \\ PIER PAOLO PARNIGOTTO ${ }^{2}$ and CLAUDIO GRANDI ${ }^{1}$ \\ ${ }^{1}$ Department of Pharmaceutical and Pharmacological Sciences, University of Padua, Padua 35131; \\ ${ }^{2}$ Foundation for Biology and Regenerative Medicine, TES-Tissue Engineering and Signaling (ONLUS), Padua 35131; \\ ${ }^{3}$ Department of Neuroradiology, Hospital of Rovigo, Rovigo 45100; ${ }^{4}$ Department of Plastic and Reconstructive Surgery, \\ University of Padua, Padua 35131, Italy
}

Received July 25, 2013; Accepted February 27, 2014

DOI: $10.3892 / \mathrm{mmr} .2014 .2348$

\begin{abstract}
The present study designed and developed blood vessel substitutes (BVSs) composed of polyvinyl alcohol (PVA) cryogels. The in vitro results demonstrated that the coating of the polymer with lyophilized decellularized vascular matrix (DVM) greatly enhanced the adhesion of human umbilical vein endothelial cells (HUVECs). However, when PVA/DVM BVSs were implanted into the abdominal aorta of Sprague-Dawley rats, DVM was identified as a highly thrombogenic surface resulting in the mortality of all animals 3-4 days after surgery. By contrast, all rats implanted with PVA survived and were sacrificed after 12 months. The luminal surface of the explanted grafts was completely covered by endothelial cells and the inner diameter was similar to that of the original vessel. In conclusion, the present study indicated that PVA may be considered as a promising biomaterial for the fabrication of artificial vessels.
\end{abstract}

\section{Introduction}

At present, cardiovascular diseases are the leading cause of mortality in Western countries and patients with cardiovascular disease often require vascular reconstruction (1). To replace malfunctioning or diseased blood vessels, several reconstructive procedures have been developed, including the transfer of healthy tissue from one site of the donor to another site and the use of tissue engineered blood vessels (1). Although high-flow vessels with a large diameter $(>6 \mathrm{~mm})$ are successful, autografts, allografts or synthetic prosthetic grafts are unable to meet the clinical demand,

Correspondence to: Professor Claudio Grandi, Department of Pharmaceutical and Pharmacological Sciences, University of Padua, Via Marzolo 5, Padua I-35131, Italy

E-mail: claudio.grandi@unipd.it

Key words: polyvinyl alcohol, vascular tissue engineering, scaffold, decellularized matrix, vascular graft particularly in low-flow small-diameter vascular grafts, which are often associated with thrombosis, aneurysm formation, calcification and severe inflammatory reactions (2). Thus, the development of an effective vascular grafts, characterized by a non-thrombogenic surface and possessing biomechanical properties matching those of native vessels, has become one of the main targets of tissue engineering. Several polymers have been used to obtain blood vessel substitutes (BVSs): Poly(dimethylsiloxane), poly(caprolactone), poly(methyl methacrylate), poly-L-lactic acid (PLLA), polyglycolic acid and poly(glycerol sebacate) (3).

In this context, polyvinyl alcohol (PVA), a linear synthetic polymer derived from the hydrolysis of polyvinyl acetate, has not yet been investigated. As a consequence of its water solubility, PVA must be cross-linked by either physical or chemical methods in order to form stable hydrogels whose mechanical properties depend on the degree of the crosslinks which, simultaneously, determine the water uptake and diffusional features of the biomaterial (4). Due to its biocompatibility and swelling properties, PVA has been approved by the Food and Drug administration and is widely used to produce medical devices, including drug delivery devices, hemodialysis membranes and soft contact lenses (5). Furthermore, PVA hydrogels have been evaluated as scaffolds for tissue engineering purposes, particularly as soft tissue substitutes (4). Indeed, the three-dimensional network of polymer chains allows the removal of wastes and the diffusion of nutrients and oxygen, which are required for cell survival.

Previous studies have demonstrated that PVA hydrogels possess mechanical properties similar to those of porcine aortas (6) and human arteries $(7,8)$, thus suggesting their suitability for the fabrication of BVSs. However, the highly hydrophilic nature of PVA hinders cell adhesion, which has a major role in the outcome of vascular grafts. Moreover, the in vivo formation of an endothelial lining on the biomaterial is required to avoid platelet adhesion and ultimately dictates the success of the reconstructive surgery. Consequently, several approaches have been applied to improve endothelial cell (EC) adhesion on PVA. Liu et al (9) blended PVA with various natural macromolecules and demonstrated that gelatin increased in vitro EC proliferation. Furthermore, Vrana et al (10) verified 
the influence of shear stress conditions on proliferation and the expression of adhesion molecules of ECs that were cultured on PVA/gelatin cryogels. Enhancement of cell adhesion was also achieved by blending PVA with chitosan (11), a positively charged polysaccharide derived from shellfish and through polyesterification of PVA with citric acid (12). However, few in vivo studies have been performed to verify the effectiveness of these biomaterials as vascular grafts $(13,14)$.

In a previous study conducted by our group (15), a three-layered vessel substitute (TLVS) was developed, which was composed of a core of PLLA coated on both sides with a homogenate of decellularized bovine aorta. TLVS possessed good mechanical properties and the extracellular matrix (ECM) components improved the adhesion and growth of human ECs and smooth muscle cells.

The present study aimed to design and develop BVSs composed of either PVA or PVA coated with a lyophilized decellularized vascular matrix (DVM). First of all, human umbilical vein endothelial cell (HUVEC) adhesion and proliferation on BVSs were assessed in vitro. BVSs were implanted into the abdominal aorta of Sprague-Dawley rats and the outcomes of the reconstructive surgery were evaluated after 12 months.

\section{Materials and methods}

Reagents. All the chemicals and reagents used in the present study were obtained from Sigma-Aldrich (St. Louis, MO, USA), with the following exceptions: Phosphate-buffered saline (PBS) tablets were purchased from Gibco Invitrogen Corp. (Paisley, UK), sodium chloride from Fluka Chemie AG (Basel, Switzerland), Vectashield Mounting medium for fluorescence with DAPI from Vector Laboratories, Inc. (Burlingame, CA, USA), Movat pentachrome staining kit from Bio-Optica (Milano, Italy), Milli-Q ultrapure water from the Milli-Q Integral Water Purification System (Millipore, Bedford, MA, USA), the mouse monoclonal anti-von Willebrand Factor (vWF) antibody from Abcam (Cambridge, UK), the goat anti-mouse immunoglobulin (IgG)-fluorescein isothiocyanate (FITC; SC-2010) antibody from Santa Cruz Biotechnology, Inc. (Santa Cruz, CA, USA), the CellTiter $96{ }^{\circledR}$ AQueous One Solution Cell Proliferation Assay from Promega Corporation (Madison, WI, USA), the Endothelial Cell Growth Medium $\mathrm{MV}_{2}$ from PromoCell $\mathrm{GmbH}$ (Heidelberg, Germany).

Fabrication of PVA and PVA/DVM BVSs. Two types of BVSs were fabricated: one was composed of only PVA and one was composed of a lyophilized decellularized vessel matrix arranged as internal concentric layers to PVA.

PVA [molecular weight (MW) 146,000-186,000 g/mol; $16 \mathrm{~g}$ ] and polyethylene glycol (PEG, MW 1,000 g/mol; $160 \mathrm{mg}$ ) were dissolved into $84 \mathrm{ml}$ distilled water at $95-100^{\circ} \mathrm{C}$ for $\sim 2 \mathrm{~h}$. To fabricate PVA BVSs, the polymer solution was aspirated into specific tubular moulds ( $2 \mathrm{~mm}$ in diameter) and then stainless steel rods (10 $\mathrm{cm}$ in length and $1 \mathrm{~mm}$ in diameter) were fixed concentrically into the moulds.

To produce PVA/DVM BVSs, tibial arteries were harvested from calves. Following the removal of the surrounding soft tissues, vessels were extensively washed and decellularized using the modified Meezan's method as previously described (16). A single decellularization cycle consisted of three steps: i) treatment with distilled water for $72 \mathrm{~h}$ at $4^{\circ} \mathrm{C}$; ii) incubation in $4 \%$ sodium deoxycholate solution for $4 \mathrm{~h}$ at room temperature; and after washing, iii) treatment with 2,000 KU DNase-I in $1 \mathrm{M} \mathrm{NaCl}$ solution for $2 \mathrm{~h}$ at room temperature. The decellularization protocol was repeated four times as previously described (17). Decellularized vessels $(1 \mathrm{~g})$ were cut into small pieces and homogenized in $15 \mathrm{ml}$ of cold $1.6 \mathrm{M}$ acetic acid using an IKA T 10 Basic Ultra Turrax homogenizer (IKA ${ }^{\circledR}$-Werke GmbH \& Co. KG, Staufen, Germany) (15). A stainless steel rod (10 cm in length and $1 \mathrm{~mm}$ in diameter) was pre-cooled at $-70^{\circ} \mathrm{C}$ and dipped in decellularized vessel homogenate several times until the layer deposited reached a thickness of $\sim 1 \mathrm{~mm}$. The deposited material was then lyophilized and the stainless steel mandrel, covered by a spongy and dry layer of DVM, was dipped into a $16 \%$ PVA solution and prepared as described above.

Both types of BVS were cross-linked through freeze/thaw cycles. Moulds were put in a cold bath and frozen at $-20^{\circ} \mathrm{C}$ for $12 \mathrm{~h}$, and then stored at $-2 /-5^{\circ} \mathrm{C}$ for $12 \mathrm{~h}$. The two last steps were repeated three times. BVSs were removed from the moulds and sterilized in $80 \%$ (v/v) ethanol solution for $4 \mathrm{~h}$ under UV light in a sterile hood. Scaffolds were then stored in PBS containing a $1 \%$ antibiotic and antimycotic solution at $4^{\circ} \mathrm{C}$ until use.

Cell cultures. Primary cultures of HUVECs were obtained by enzymatic digestion of the umbilical vein endothelial layer with a $0.1 \%$ collagenase IV solution. The cells were seeded on Petri dishes previously coated with $1 \mu \mathrm{g} / \mathrm{ml}$ fibronectin and cultured with Endothelial Cell Growth Medium $\mathrm{MV}_{2}$ supplemented with $5 \%$ fetal calf serum, $1 \mu \mathrm{g} / \mathrm{ml}$ ascorbic acid, $10 \mathrm{ng} / \mathrm{ml}$ human fibroblastic growth factor-2, $5 \mathrm{ng} / \mathrm{ml}$ human epidermal growth factor, $0.2 \mu \mathrm{g} / \mathrm{ml}$ hydrocortisone, $20 \mathrm{ng} / \mathrm{ml} \mathrm{R}^{3}$-insulin-like growth factor-1, $0.5 \mathrm{ng} / \mathrm{ml}$ vascular endothelial growth factor (endothelial $\mathrm{MV}_{2}$ medium kit) and $1 \%$ antibiotic solution, containing $10 \mathrm{ng} / \mathrm{ml}$ streptomycin sulfate, $250 \mathrm{ng} / \mathrm{ml}$ amphotericin-B and $100 \mathrm{U} / \mathrm{ml}$ penicillin. Cultures were incubated at $37^{\circ} \mathrm{C}$ in a humidified atmosphere. HUVECs were used until the fourth passage and harvested at $80 \%$ confluence.

Proliferation assay. The (3-(4,5-dimethylthiazol-2-yl)-5-(3-carb oxymethoxyphenyl)-2-(4-sulfophenyl)-2H-tetrazolium inner salt (MTS) dye assay was used to evaluate cell proliferation. HUVECs $\left(3 \times 10^{5}\right.$ cells $\left./ \mathrm{cm}^{2}\right)$ were previously seeded on either PVA or PVA/DVM BVSs and deposited onto 12-well plates. Cultures grown on tissue culture-treated polystyrene plates were used as a positive control. Twenty-four, 48 and $92 \mathrm{~h}$ after seeding, cell proliferation was determined using the CellTiter 96 ${ }^{\circledR}$ AQueous One Solution Cell Proliferation assay according to the manufacturer's instructions. Briefly, cells were treated with $10 \%$ MTS for $4 \mathrm{~h}$. Optical density of purple formazan produced in living cells was measured at $490 \mathrm{~nm}$ using a Microplate autoreader EL 13 (Bio-Tek Instruments, Winooski, VT, USA). The means of three experiments were expressed as the number of cells. The linearity of absorbance of formazan ranging from $3 \times 10^{3}$ to $2 \times 10^{4}$ cells was established by determining the linear coefficient (0.986). All results were expressed as the mean \pm standard deviation 

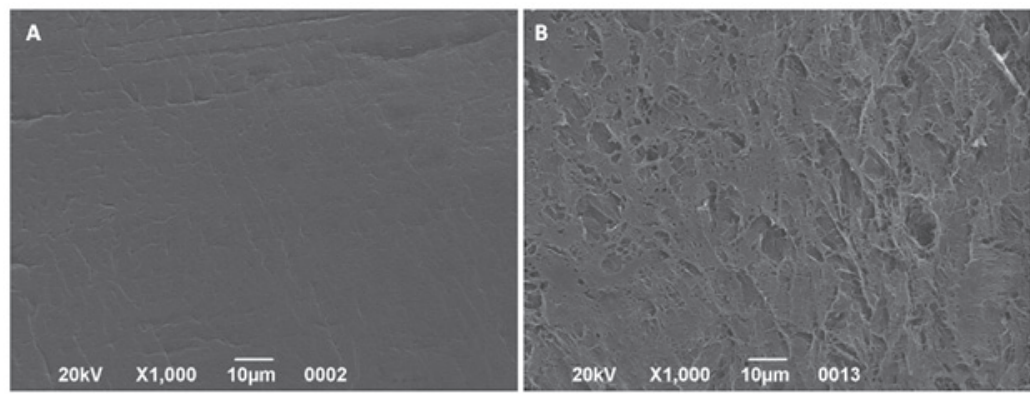

Figure 1. Scanning electron microscopy micrographs of the (A) external side and (B) internal side of polyvinyl alcohol/decellularized vascular matrix blood vessel substitutes.
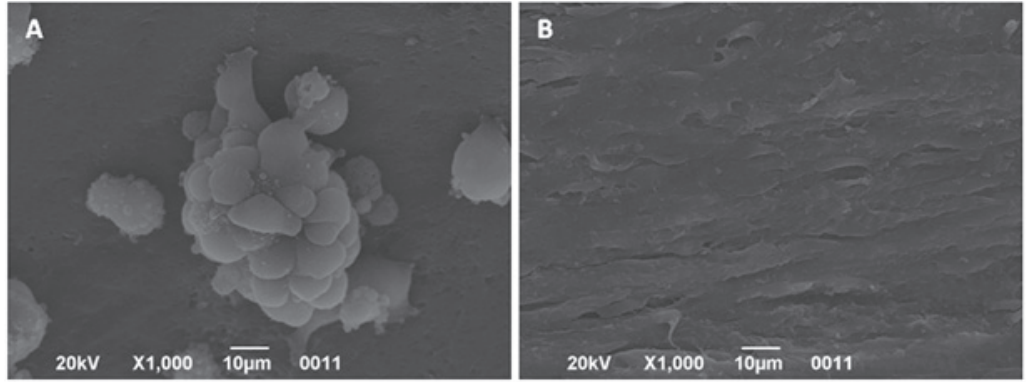

C

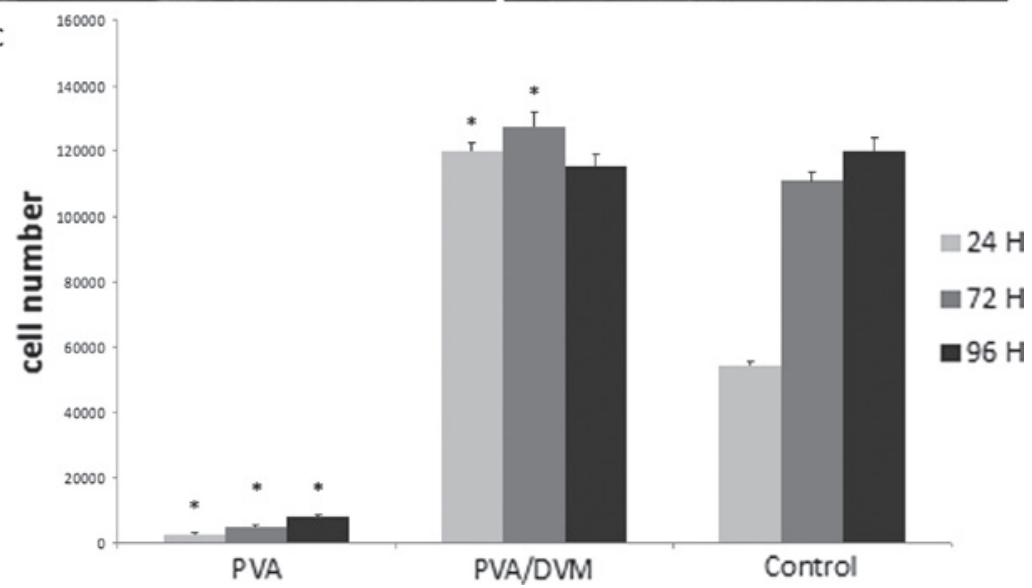

Figure 2. Scanning electron microscopy micrographs of HUVEC cultures on (A) PVA and (B) PVA/DVM $96 \mathrm{~h}$ after seeding. (C) HUVEC proliferation at 24 (light grey), 72 (dark grey) and $96 \mathrm{~h}$ (black) after seeding on PVA, PVA/DVM and tissue culture-treated plates (control). Results are expressed as the cell number as the mean \pm standard deviation of at least three independent experiments. "P $<0.05$ vs. control cultures, Student's t-test. HUVEC, human umbilical vein endothelial cell; PVA, polyvinyl alcohol; DVM, decellularized vascular matrix.

of three separate experiments. Statistical comparison was performed by analysis of variance, followed by the Student's t-test. Alternatively, cultures were fixed with $10 \%$ formalin. Samples were then examined after critical-point drying and gold sputtering was applied prior to scanning electron microscopy (SEM; Stereoscan-205 S; Cambridge Instruments, Cambridge, MA, USA).

In vivo experiments. BVSs were inserted to repair a surgically created defect in the abdominal aorta of 14 male Sprague-Dawley rats weighing 250-300 g and aged 6 months. Animals were divided in two groups according to the implanted graft: Group $1(n=7)$ received PVA BVSs and Group $2(n=7)$ received PVA/DVM BVSs. The protocol was approved by the Institutional Animal Care Committee of the University of Padova (Padova, Italy). Under halothane anesthesia, the abdominal area was shaved and aseptically prepared using povidone-iodine (Betadine). The muscles were exposed with a $3 \mathrm{~cm}$ abdominal incision and the abdominal aorta was exposed and isolated. After clamping the vessel, a segment of aorta (1 cm in length) was excised and the scaffold $(2 \mathrm{~mm}$ in diameter and $2 \mathrm{~cm}$ in length) was anastomized proximally and distally end-to-end using continuous 10.0 polypropylene sutures. No anticoagulants and antibiotics were administered. The animals implanted with PVA/DVM BVSs died 3-4 days after surgery, whereas the rats implanted with PVA BVSs were sacrificed after 12 months. The implants were recovered and each sample was divided into two pieces: one was frozen in liquid nitrogen and cut into $10-\mu \mathrm{m}$ thick cryosections for histological analysis, one was fixed with $10 \%$ formalin for SEM and processed as described above.

Cryostat sections were fixed in cold acetone for $5 \mathrm{~min}$. The sections were then stained using the Movat pentachrome stain kit according to the manufacturer's instructions. Samples 

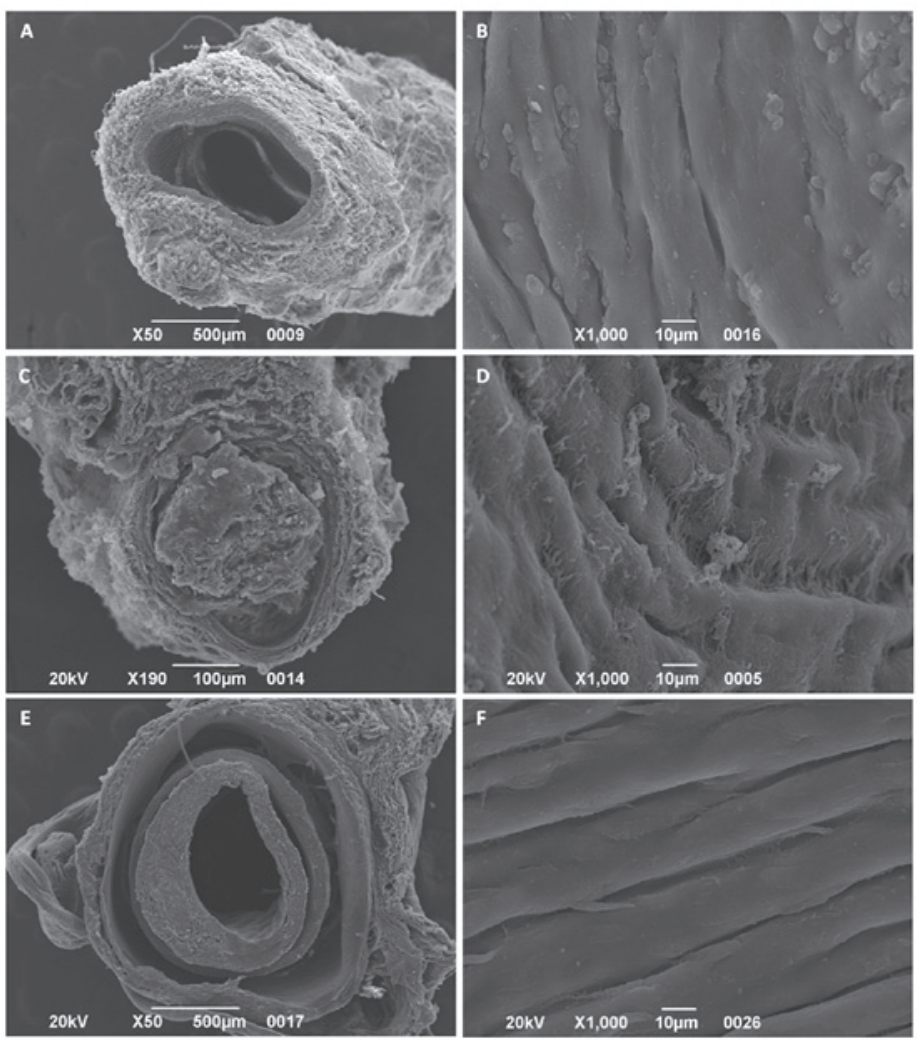

Figure 3. Scanning electron microscopy micrographs of (A and B) native abdominal aorta, (C and D) PVA/decellurized vascular matrix, and (E and F) PVA implants. (A, C and E) Cross-sections; (B, D and F) luminal sides. PVA, polyvinyl alcohol.
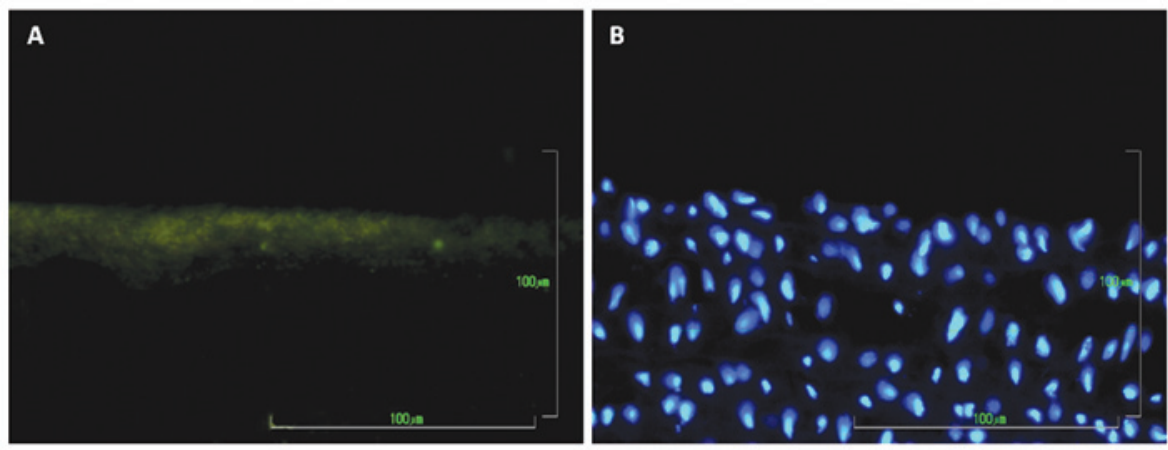

Figure 4. Immunofluorescence of polyvinyl alcohol implants stained with (A) anti-von Willebrand Factor and (B) DAPI (magnification, x200).

were examined under an optical microscope (Leica DM2000; Leica Microsystems, Wetzlar, Germany). Alternatively, aspecific sites were blocked with $10 \%$ goat serum in PBS for $45 \mathrm{~min}$ at room temperature. Samples were incubated with monoclonal anti-vWF antibody (1:100 in 3\% goat serum) at $4^{\circ} \mathrm{C}$ overnight and then incubated with the secondary antibody, goat anti-mouse IgG-FITC (1:200 in 1.5\% goat serum) for $1 \mathrm{~h}$ at room temperature. Slices were mounted with mounting medium with DAPI. Negative controls were obtained by omitting the primary antibody.

\section{Results}

Morphological analysis. Fig. 1 shows the structure of PVA/DVM BVSs. The external side (Fig. 1A), composed of PVA, appeared smooth, whereas the internal side (Fig. 2B), coated with lyophilized DVM, exhibited a porous structure and bundles of crimped fibers.

Only a few clusters of rounded cells were visible on PVA (Fig. 2A) $96 \mathrm{~h}$ after seeding. By contrast, HUVECs formed a confluent monolayer on the internal side of PVA/DVM after 24 h (Fig. 2B).

Cell proliferation assay. The results of the cell proliferation assays supported the findings of the morphological analysis (Fig. 2C). The cell number in cultures on PVA/DVM did not increase from 24 to $96 \mathrm{~h}$ and were significantly higher than those determined in the corresponding control cultures grown on tissue culture-treated polystyrene plates. By contrast, the number of cells detected on PVA was significantly lower in comparison to those counted in the PVA/DVM and the control groups. 

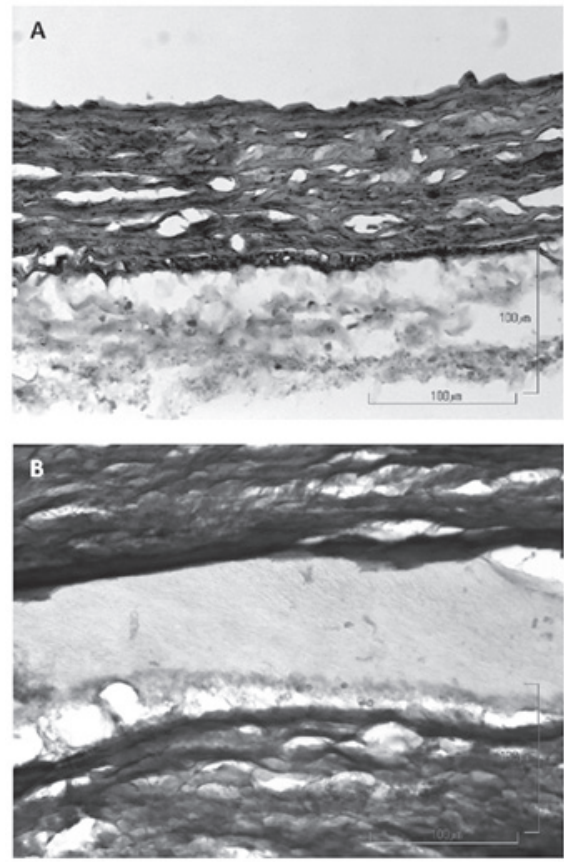

Figure 5. Movat staining of the (A) native abdominal aorta and (B) polyvinyl alcohol cryogels (magnification, x200).

SEM analysis and immunofluorescence. Sprague-Dawley rats received either PVA or PVA/DVM BVSs as abdominal aorta interposition grafts $(2 \mathrm{~cm})$. All animals who received PVA/DVM BVSs died 3-4 days after surgery. As shown in Fig. 3C, grafts were totally or partially occluded by thrombi and the luminal surface presented a network of fibrin fibres (Fig. 3D). By contrast, all rats implanted with PVA BVSs survived surgery without signs of infection or implant rejection. After 12 months, the outcome was evaluated by means of SEM, immunofluorescence and Movat staining. PVA grafts (Fig. 3E) were patent and both sides were wrapped by regenerating tissue continuous to that of native vessels. Although the implants appeared thicker than the wall of the host aorta (Fig. 3A), the inner diameters of all PVA grafts were similar to those of the original vessels. Furthermore, compared with the native artery (Fig. 3B), the luminal surface of the grafts was completely covered by cells orientated along the blood flow in longitudinal beamed structures (Fig. 3F). These cells were immunoreactive to anti-vWF antibody, confirming their endothelial phenotype (Fig. 4A). Movat staining demonstrated that the tissue layer covering the luminal side of PVA (Fig. 5B) resembled the structure of the native aorta (Fig. 5A). However, no cells were visible inside the polymer.

\section{Discussion}

To date, the development of functionally and biologically compatible BVSs has gained increasing attention due to the augmented morbidity of cardiovascular diseases in Western countries. Thus, the fabrication of suitable vascular grafts is one of the main targets of tissue engineering. Since Weinberg and Bell (18) reported the construction of blood vessels using collagen, cultured bovine aortic ECs, smooth muscle cells and adventitial fibroblasts in vitro, considerable progress in this field has been achieved. Several approaches have emerged in the last two decades, including ECM protein and nanostructured synthetic polymer-based grafts and scaffold-free cell transplantable constructs (3). Although venous grafts have entered clinical trials (19), the achievement of suitable arterial substitutes, particularly those of small diameters ( $<6 \mathrm{~mm}$ ) for coronary artery bypass procedures or peripheral arterial revascularization, remains a challenge. The ideal BVS must possess mechanical properties that are able to sustain a high-pressured pulsatile blood flow. Furthermore, BVSs must promote cell growth, inhibit thrombogenesis, avoid inflammation and neointimal proliferation.

In this context, previous studies have reported that PVA hydrogels possess mechanical properties resembling those of human arteries $(7,8)$. King et al $(8)$ demonstrated that this biomaterial can be used as a vessel wall mimicking material in anatomically realistic flow phantoms. In vitro and in vivo experiments have also shown that the PVA surfaces were athrombogenic $(13,14)$. However, PVA does not warrant cell adhesion and proliferation. Herein, to improve the compatibility with vascular cells, the internal surface of tubular devices composed of PVA cryogels were coated with lyophilized DVM. As expected, the in vitro results of the present study showed that the PVA surface was unable to support cell adhesion and growth. By contrast, the presence of ECM macromolecules greatly enhanced the adhesion of HUVECs, allowing the formation of a confluent monolayer $24 \mathrm{~h}$ after seeding. A previous study conducted by our group demonstrated that the DVM contained types I and VI collagen, which mediated cell adhesion through the RGD motif (15). Furthermore, the decellularization process may have maintained growth and angiogenic factors, including basic fibroblast growth factor and transforming growth factor- $\beta$, as previously detected in skeletal muscle acellular matrices $(20,21)$. Thus, the presence of tissue-specific proteins and growth factors may make DVM a more permissive environment for vascular cells, with regard to the addition of single bioactive molecules to the biomaterials $(22,23)$.

In the present study, when PVA/DVM BVSs were implanted into the abdominal aorta of Sprague-Dawley rats, DVM was found to be a highly thrombogenic surface, which subsequently resulted in the mortality of all animals 3-4 days after surgery. Obstruction of the grafts occurred due to the formation of a fibrin network on the luminal side and activation of the coagulative pathway. These results clearly indicated the need to mask ECM proteins in order to avoid platelet adhesion and thrombi formation. In this context, recellularizing scaffolds with host ECs prior to implantation or treatment of the inner lining with heparin may be a useful approach (24). By contrast, all animals receiving PVA BVSs survived until the time of sacrification (12 months). Miyake et al (14) reported that the patency of PVA tubes implanted in the carotid artery of rats was $80 \%$ after 1 week and $70 \%$ after 1 month. To make thromboresistant woven Dacron artificial vessels, Tamura et al (13) coated them with either PVA-silica or heparinized PVA-silica. One year after implantation into the abdominal aorta of adult mongrel dogs, the percentage of patent grafts ranged from $\sim 57$ to $67 \%$. Herein, although neither anticoagulants nor fibrinolitic agents were administered, all PVA BVSs remained patent. Microscopic examination identified a continuous EC coverage 
of the luminal surface of the explanted PVA grafts whose inner diameter was similar to that of the native vessel. Despite the lack of cells inside the polymer, PVA acted as a guide for tissue regeneration and the host tissue was grown on both sides of the graft, which resulted in a neovascular wall whose architecture resembled that of the original vessel.

In conclusion, the present study demonstrated that an effective and long-term replacement of tracts of the abdominal aorta can be achieved using tubes composed of PVA cryogels. This biomaterial, coupling biocompatible and non-thrombogenic features with the capability to withstand high-flow rates, may be considered as a promising tool for the fabrication of artificial vessels. Further studies are required to monitor the local tissue responses of the host at time-points prior to 12 months.

\section{Acknowledgements}

The authors would like to thank Dr Ilenia Zanusso for advice on the micrographs.

\section{References}

1. Nemeno-Guanzon JG, Lee S, Berg JR, Jo YH, Yeo JE, Nam BM, Koh YG and Lee JI: Trends in tissue engineering for blood vessels. J Biomed Biotechnol 2012: 956345, 2012.

2. Schimidt CE and Baier JM: Acellular vascular tissues: natural biomaterials for tissue repair and tissue engineering. Biomaterials 21: 2215-2231, 2000.

3. Hung HS, Chen HC, Tsai CH and Lin SZ: Novel approach by nanobiomaterials in vascular tissue engineering. Cell Transplant 20: 63-70, 2011.

4. Baker MI, Walsh SP, Schwartz Z and Boyan BD: A review of polyvinyl alcohol and its uses in cartilage and orthopedic applications. J Biomed Mater Res B Appl Biomater 100: 1451-1457, 2012.

5. Cascone MG, Barbani N, Cristallini C, Giusti P, Ciardelli G and Lazzeri L: Bioartificial polymeric materials based on polysaccharides. J Biomater Sci Polym 12: 267-281, 2001.

6. Chu KC and Rutt BK: Polyvinyl alcohol cryogel: an ideal phantom material for MR studies of arterial flow and elasticity. Magn Reson Med 37: 314-319, 1997.

7. O'Flynn PM, Roche ET and Pandit AS: Generating an ex vivo vascular model. ASAIO J 51: 426-433, 2005.

8. King DM, Moran CM, McNamara JD, Fagan AJ and Browne JE: Development of a vessel mimicking material for use in anatomically realistic Doppler flow phantoms. Ultrasound Med Biol 37: 813-826, 2011.

9. Liu Y, Vrana NE, Cahill PA and McGuinness GB: Physically crosslinked composite hydrogels of PVA with natural macromolecules: structure, mechanical properties, and endothelial cell compatibility. J Biomed Mater Res B Appl Biomater 90: 492-502, 2009 .
10. Vrana NE, Cahill PA and McGuinnes GB: Endothelization of PVA/gelatin cryogels for vascular tissue engineering: effect of disturbed shear stress conditions. J Biomed Mater Res A 94: 1080-1090, 2010.

11. Mathews DT, Birney YA, Cahill PA and McGuinness GB: Vascular cell viability on polyvinyl alcohol hydrogels modified with water-soluble and -insoluble chitosan. J Biomed Mater Res B Appl Biomater 84: 531-540, 2008.

12. Thomas LV, Arun U, Remya S and Nair PD: A biodegradable and biocompatible PVA-citric acid polyester with potential applications as matrix for vascular tissue engineering. J Mater Sci Mater Med 20: S259-S269, 2009.

13. Tamura K, Mizuno H, Okada K, Katoh H, Hitomi S, Teramatsu T, Shimizu Y and Hino T: Experimental application of polyvinyl alcohol-silica for small artificial vessels. Biomater Med Devices Artif Organs 13: 133-152, 1985-1986.

14. Miyake H, Handa H, Yonekawa Y, Taki W, Naruo Y, Yamagata S, Ikada Y, Iwata H and Suzuki M: New small-caliber antithrombotic vascular prosthesis: experimental study. Microsurgery 5: 144-150, 1984.

15. Grandi C, Martorina F, Lora S, Dalzoppo D, Amistà P, Sartore L, Di Liddo R, Conconi MT and Parnigotto PP: ECM-based triple layered scaffolds for vascular tissue engineering. Int J Mol Med 28: 947-952, 2011.

16. Meezan E, Hjelle JT, Brendel K and Carlson EC: A simple, versatile, non disruptive method for the isolation of morphologically and chemically pure basement membranes from several tissues. Life Sci 17: 1721-1732, 1975.

17. Grandi C, Baiguera S, Martorina F, Lora S, Amistà P, Dalzoppo D, Del Gaudio C, Bianco A, Di Liddo R, Conconi MT and Parnigotto PP: Decellularized bovine reinforced vessels for small-diameter tissue-engineered vascular grafts. Int J Mol Med 28: 315-325, 2011

18. Weinberg CB and Bell E: A blood vessel model constructed from collagen and cultured vascular cells. Science 231: 397-400, 1986

19. Udelsman BV, Maxfield MW and Breuer CK: Tissue engineering of blood vessels in cardiovascular disease: moving towards clinical translation. Heart 99: 454-460, 2013.

20. De Coppi P, Bellini S, Conconi MT, Sabatti M, Simonato E, Gamba PG, Nussdorfer GG and Parnigotto PP: Myoblast-acellular skeletal muscle matrix constructs guarantee a long-term repair of experimental full-thickness abdominal wall defects. Tissue Eng 12: 1929-1936, 2006.

21. Conconi MT, Bellini S, Teoli D, de Coppi P, Ribatti D, Nico B, Simonato E, Gamba PG, Nussdorfer GG, Morpurgo M and Parnigotto PP: In vitro and in vivo evaluation of acellular diaphragmatic matrices seeded with muscle precursors cells and coated with VEGF silica gels to repair muscle defect of the diaphragm. J Biomed Mater Res A 89: 304-316, 2009.

22. Ju YM, Choi JS, Atala A, Yoo JJ and Lee SJ: Bilayered scaffold for engineering cellularized blood vessels. Biomaterials 31 : 4313-4321, 2010

23. Stephan S, Ball SG, Williamson M, Bax DV, Lomas A, Shuttleworth CA and Kielty CM: Cell-matrix biology in vascular tissue engineering. J Anat 209: 495-502, 2006.

24. Thomas LV, Lekshmi V and Nair PD: Tissue engineered vascular grafts - preclinical aspects. Int J Cardiol 167: 1091-1100, 2013. 